\title{
Endocan and Non-Dipping Circadian Pattern in Newly Diagnosed Essential Hypertension
}

Tolga Çimen, MD¹, Murat Bilgin, MD¹, Ahmet Akyel, MD¹, Mehmet Ali Felekoğlu, MD¹, Ali Nallbani, MD', Şeyda Özdemir, MD², Gönül Erden, MD², Alpaslan Öztürk, MD², Mehmet Doğan, MD', and Ekrem Yeter, MD

${ }^{1}$ Department of Cardiology, Dışkapı Yıldırım Beyazıt Training and Research Hospital, Ankara, ${ }^{2}$ Department of Clinical Biochemistry, Dışkapı Yıldırım Beyazıt Training and Research Hospital, Ankara, Turkey

Background and Objectives: Non-dipper hypertension is frequently accompanied by endothelial dysfunction and activation. Previous studies suggested that endocan may be a novel endothelial dysfunction marker. This study aims to investigate the association between circadian blood pressure (BP) pattern and plasma endocan levels together with high-sensitivity C-reactive protein (hsCRP) in patients with newly diagnosed untreated hypertension.

Subjects and Methods: Twenty-four hour ambulatory blood pressure monitoring was recorded in 35 dipper, 35 non-dipper hypertensives and 35 healthy controls. Endocan levels were measured by enzyme-linked immunosorbent assay. Serum levels of hsCRP were also recorded. Results: Despite similar daytime and 24-hour average BP values between dippers and non-dippers, statistically significant high nocturnal BP was accompanied by a non-dipping pattern (Systolic BP: $132 \pm 9$ vs. $147 \pm 11 \mathrm{mmHg}$; Distolic BP: $80 \pm 7$ vs. $91 \pm 9 \mathrm{mmHg}$, respectively, $p<0.001$ for both). Non-dipper patients demonstrated higher endocan levels compared to dippers and normotensives (367 (193-844) pg/mL, 254 $(182-512) \mathrm{pg} / \mathrm{mL}$ and 237 (141-314) $\mathrm{pg} / \mathrm{mL}$, respectively, $\mathrm{p}<0.001)$. HsCRP levels were significantly higher in non-dippers than the other groups ( $p=0.013)$. In a multivariate logistic regression analysis, endocan $(p=0.021)$ and hsCRP ( $p=0.044)$ were independently associated with a non-dipping pattern.

Conclusion: Elevated endocan levels were found in non-dipper groups. Endocan and hsCRP were found to be independently associated with a non-dipping pattern. We suggest that elevated levels of endocan in non-dipper hypertensive patients might be associated with a longer duration of exposure to high BP. These results point to the possible future role of endocan in selection of hypertensive patients at higher risk or target organ damage. (Korean Circ J 2016;46(6):827-833)

KEY WORDS: Blood pressure monitoring, ambulatory; Hypertension; Dipping pattern; Endocan.

\section{Introduction}

Hypertension (HT) is one of the most prominent risk factors for adverse cardiovascular (CV) events.") High blood pressure (BP) is

Received: December 4, 2015

Revision Received: January 27, 2016

Accepted: April 5, 2016

Correspondence: Tolga Çimen, Dışkapı Yıldııım Beyazıt Training and Research Hospital, Cardiology Department, Altındağ, Ankara, Turkey Tel: 90-312-5962933, Fax: 90-312-3186690

E-mail:drtolgacim@hotmail.com

- The authors have no financial conflicts of interest.

This is an Open Access article distributed under the terms of the Creative Commons Attribution Non-Commercial License (http://creativecommons. org/licenses/by-nc/3.0) which permits unrestricted non-commercial use, distribution, and reproduction in any medium, provided the original work is properly cited. frequently accompanied by endothelial dysfunction, increased oxidative stress and vascular complications. ${ }^{2)}$ Twenty-four hour ambulatory blood pressure monitoring (ABPM) is useful in the diagnosis of $\mathrm{HT}$ and assessment of BP regulation and variability. Many studies have shown that assessing the circadian BP profile is more predictive than office $B P$ readings in estimating cardiovascular risk. ${ }^{3)}$ In most of the subjects nocturnal BP values are considerably lower than daytime BP values. A non-dipping BP profile is usually defined as a nocturnal BP fall of less than 10\%. The attenuation of a nocturnal BP decline should be regarded as abnormal and is associated with increased CV events. ${ }^{4)}$ In addition, target organ damage is more pronounced in non-dippers such as an increased mass of the left ventricle, ${ }^{5)}$ carotid artery disease. ${ }^{6)}$ Release of endothelial nitric oxide is diminished in patients with HT resulting in decreased endothelium-dependent vasodilation which constitutes the first step of development of atherosclerosis. It was 
shown that endothelium-dependent vasodilation is much more impaired in non-dippers.?

Endothelial cell-specific molecule-1 (endocan) is in structure of dermatan sulfate pretoglycan and released actively by endothelial cells. ${ }^{8}$ It has been shown to compete with intercellular adhesion molecule-1 (ICAM-1) for leukocyte function-associated antigen (LFA-1).9) Blood levels are elevated in cases with endothelial activation such as inflammation and tumoral diseases. ${ }^{10)}$ It is considered to have a role in the cellular adhesion and angiogenesis process. ${ }^{11)}$ Elevated blood levels of endocan in overt and subclinical atherosclerotic diseases makes it a surrogate marker of endothelial activation and dysfunction. ${ }^{1213)}$ Herein, we investigated the association between a circadian BP pattern and plasma endocan levels together with high-sensitivity C-reactive protein (hsCRP) in patients with newly diagnosed untreated essential HT.

\section{Subjects and Methods}

\section{Patients and groups}

In the current study, we prospectively screened 88 subjects. Seventy hypertensive patients aged between 18 and 75 years who applied to the cardiology polyclinic were selected by excluding 1 patient suspicious for adrenal adenoma, 13 patients with accompanying diseases, and 4 patients taking medications with side effects. They were divided into dipper and non-dipper groups after a 24-hour ABPM assessment. Office blood pressures of all hypertensive patients were $\geq 140$ and/or $\geq 90 \mathrm{mmHg}$ (the average of $\geq 2$ proper readings measured on at least two visits). In addition, a control group is constituted with 35 healthy normotensive participants. Patients in the study were newly diagnosed patients with no use of previous antihypertensive therapy. Exclusion criteria were as follows: patients who reject participation to the study, patients with secondary HT, diabetes, systolic dysfunction of left ventricle (ejection fraction $<50 \%$ ), atrial fibrillation, history of coronary artery disease or angina, moderate to severe valvular diseases, malignancy, known chronic obstructive pulmonary disease and obstructive sleep apnea, moderate to severe renal (estimated glomerular filtration rate of $<60 \mathrm{ml} / \mathrm{min}$ ) and hepatic dysfunction (presence of cirrhosis or ALT and/or AST > $3 X$ ULN and total bilirubin $>2 X U L N$ ), active inflammatory diseases, chronic use of medicine (e.g. lipid reducing and anti-inflammatory drugs, etc.), alcohol or substance addiction.

\section{Echocardiographic examination}

After detailed medical history and physical examination, echocardiography of patients were performed by an experienced cardiologist, blinded to patients' clinical and laboratory data, using a commercially available ultrasound system with a 2.5-3.5 MHz transducer (iE33, xMATRIX Echocardiography System, Phillips Medical System, Bothell, WA, USA). Left ventricular mass index (LVMI) was calculated in accordance with up to date guidelines by using the Devereux's formula. ${ }^{14)}$

\section{Measurement of ambulatory blood pressure}

A 24-hour ABPM is applied (Bravo HR ABP Sun Tech Medical Inc., Morrisville, NC, USA) to all patients following office BP measurements. Appropriate cuff sizes were selected for all patients. The device has measurements with intervals of 15 minutes in the day-time (6:00 am to 10:00 pm) and 30 minutes at night (10:00 pm to $6: 00 \mathrm{am}$ ). If less than $80 \%$ of the measurements were valid the patients were excluded. An average of 24-hour systolic BP $>130$ $\mathrm{mmHg}$ and/or diastolic BP $>80 \mathrm{mmHg}$, an average daytime systolic BP $>135 \mathrm{mmHg}$ and/or diastolic BP $>85 \mathrm{mmHg}$ or an average nighttime systolic BP $>120 \mathrm{mmHg}$ and/or diastolic BP $>70 \mathrm{mmHg}$ were diagnosed as hypertensive. ${ }^{15)}$ A non-dipper pattern was defined as a decrease of $<10 \%$ in systolic BP between the daytime and nighttime hours.

\section{Collection of blood samples and biochemical analysis}

Blood samples were taken from patients with a period of at least a 12-hour fast following ABPM. Serum levels of hsCRP, fasting blood glucose levels, creatinine levels, complete blood count and fasting serum lipid status including total cholesterol, low density lipoprotein cholesterol, high density lipoprotein cholesterol, and triglyceride levels were recorded. Blood samples were collected using EDTA as an anticoagulant and centrifuged for 15 minutes at $1500 \mathrm{~g}$ within 30 minutes of collection. Samples were stored at $-20^{\circ} \mathrm{C}$. One night before testing, samples were kept at $+4^{\circ} \mathrm{C}$ to thaw. Endocan levels were measured by ELISA according to the manufacturer's protocol (Human ESM1/Endocan PicoKine ${ }^{\mathrm{TM}}$ ELISA Kit, Boster Biological Technology Co. Ltd, Pleasanton, CA, USA).

The institutional ethics committee approved the study and written informed consent for participation in the study was obtained from all individuals. The study was conducted in accordance with the Helsinki Declaration.

\section{Statistical analysis}

Statistical analyses were conducted using the Statistical Package for the Social Sciences for Windows 21.0 (SPSS Inc., Chicago, IL, USA). The Kolmogorov-Smirnov test was used to determine whether the continuous variables were normally distributed. Normally distributed variables were given as mean \pm standard deviation, while those variables featured by non-normal distribution were given as 
median (minimum-maximum) values. Descriptive statistics are given as a percentage and absolute values. Basal characteristics were compared with a chi-square test. Where appropriate, a One-way analysis of variance (ANOVA) or Kruskal-Wallis test was used for the comparison of the 3 groups for continuous variables. Differences between subgroups were revealed using Dunn's procedure (for data without normal distribution). Data were analyzed to identify whether endocan was independently associated with the risk of non-dipper HT by using univariate logistic and multivariate logistic regression models. Univariate analyses considered the following variables: Hemoglobin, body mass index, hsCRP, age, gender, LVMI, smoking, fasting blood glucose and creatinine. Covariates with $p<0.1$ from a univariate logistic regression were included for a multivariate analysis. We performed a receiver operating characteristic (ROC) analysis to identify the most sensitive endocan cutoff level for identifying patients with non-dipper HT. A p $<0.05$ was accepted to be statistically significant. We performed a post hoc power analysis based on endocan results (effect size: $0.60, \alpha: 0.05$ ) that revealed the study power as $86 \%$ by assuming the parent distribution as a Laplace distribution.

\section{Results}

Demographic, laboratory and echocardiographic characteristics of the study population are shown in Table 1. Elevation in serum triglycerides that is close to significance level is observed in hypertensive patients. No significant difference was detected between the groups in demographic parameters beyond this, including complete blood count, lipid panel and creatinine values. It was established in the echocardiographic assessment that the left ventricle wall was thicker in the hypertensive group than the control group and higher LVMI was found in hypertensives $(p=0.001)$. There was no difference in terms of daytime and 24hour average systolic and diastolic BP readings between dipper and non-dipper groups (Table 2). Statistically significant high nocturnal BP was accompanied by non-dipping pattern $(p<0.001)$.

Endocan levels were significantly different among three groups $(p<0.001)$. In a pair wise comparison, while the non-dipping group had higher endocan levels compared to both dipper HT and control groups, there was no significant difference between endocan levels of the dipper HT and control groups ( $p>0.05$ ) (Fig. 1). Serum hsCRP levels were significantly higher in the

Table 1. Baseline characteristics of the study population

\begin{tabular}{|c|c|c|c|c|}
\hline & Control $(n=35)$ & Dipper $(n=35)$ & Non-dipper $(n=35)$ & $\mathbf{p}$ \\
\hline Age (years) & $50 \pm 6.4$ & $46.2 \pm 9.3$ & $48.4 \pm 7.2$ & 0.125 \\
\hline Men, n (\%) & $15(42)$ & $21(60)$ & $16(46)$ & 0.307 \\
\hline Body mass index $\left(\mathrm{kg} / \mathrm{m}^{2}\right)$ & $22.5(19.3-25.9)$ & $22.4(19.5-26)$ & $20.8(19,3-25.7)$ & 0.105 \\
\hline Smokers & $8(22.9)$ & $4(11.4)$ & $4(11.4)$ & 0.307 \\
\hline Total cholesterol (mg/dL) & $184.9 \pm 39.4$ & $197.4 \pm 48.6$ & $192.3 \pm 39.4$ & 0.469 \\
\hline Low density lipoprotein cholesterol (mg/dL) & $130.2 \pm 39.4$ & $132.2 \pm 40.8$ & $135.8 \pm 35.7$ & 0.826 \\
\hline High density lipoprotein cholesterol (mg/dL) & $46(30-76)$ & $44(30-72)$ & $44(30-95)$ & 0.863 \\
\hline Triglycerides (mg/dL) & $108(48-294)$ & $152(46-253)$ & $129(55-300)$ & 0.055 \\
\hline Creatinine (mg/dL) & $0.8(0.5-1.4)$ & $0.8(0.5-1.3)$ & $0.7(0.4-1.1)$ & 0.421 \\
\hline Fasting glucose (mg/dL) & $92(77-116)$ & 89 (69-109) & $85(51-112)$ & 0.183 \\
\hline Hemoglobin g/dL & $14.2 \pm 1.5$ & $14.8 \pm 1.1$ & $14.2 \pm 1.7$ & 0.135 \\
\hline White blood cell count $\left(10^{3} / \mathrm{mm}^{3}\right)$ & $8.2(4.8-11.4)$ & $8.1(4.8-10.6)$ & $8.1(5.0-14.4)$ & 0.830 \\
\hline Endocan (pg/mL) & $237(141-314)$ & $254(182-512)$ & $367(193-844)$ & $<0.001$ \\
\hline HsCRP (mg/L) & $2.9(0.1-15.0)$ & $2.2(0.1-11.0)$ & $4.0(1.0-13.84)$ & 0.013 \\
\hline \multicolumn{5}{|l|}{ Echocardiographic parameters } \\
\hline IVS (mm) & $9.9(7.3-11.8)$ & $10.5(8.1-13.2)$ & $10.8(7.9-13.5)$ & 0.019 \\
\hline Posterior wall (mm) & $9.5(7.1-11.1)$ & $10.4(8.3-12.4)$ & $10.5(7.8-12.9)$ & 0.022 \\
\hline LVEDD (mm) & $46(39-56)$ & $45(39-51)$ & $46(39-60)$ & 0.667 \\
\hline LVMI $\left(\mathrm{g} / \mathrm{m}^{2}\right)$ & $83.3(56.9-156.2)$ & $91.6(47.8-137.1)$ & $97.5(63.8-151)$ & 0.017 \\
\hline
\end{tabular}

All values are presented as mean value and SD, median value (minimum - maximum) or n (\%). HsCRP: high-sensitivity C-reactive protein, IVS: interventricular septum, LVEDD: left ventricular end diastolic diameter, LVMI: left ventricular mass index 


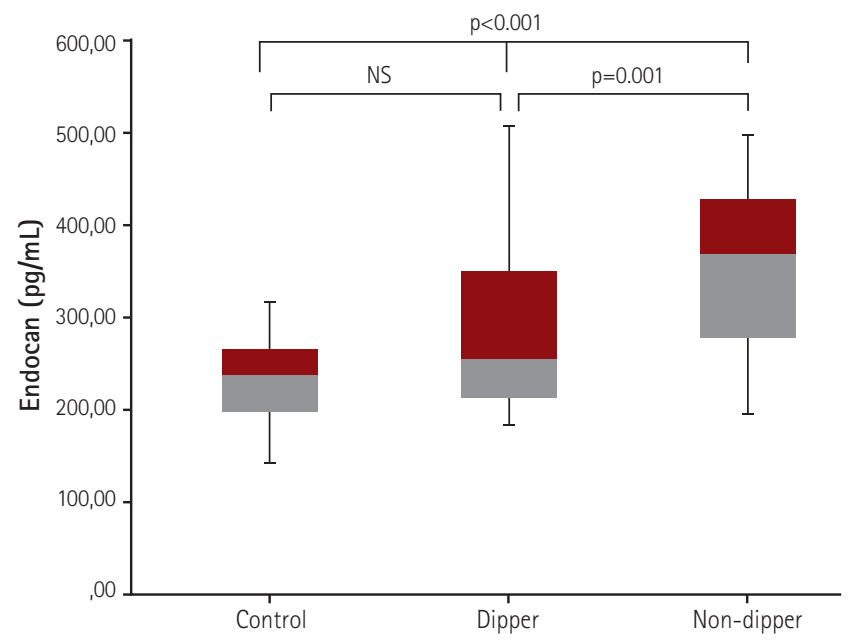

Fig. 1. Comparison of endocan levels in non-dippers compared with dippers and controls. NS: non-significant. non-dipper group than the other groups (Fig. 2). In a pairwise comparison, hsCRP levels were also significantly higher in nondippers than dippers but there were no significant differences between dippers and the control group ( $p>0.05)$. In patients with $H T$, plasma endocan levels correlated positively with hsCRP $(r=0.247, p=0.039)$. At the multivariate analysis, which was done after adjusting for potential confounding variables, higher endocan and hsCRP levels were found to be independently associated with a non-dipping pattern (Table 3). In the ROC analysis, endocan $>297 \mathrm{pg} / \mathrm{mL}$ had $68 \%$ sensitivity and $72 \%$ specificity (area under the curve $0.731, p<0.001$ ) in predicting non-dipping status (Fig. 3).

\section{Discussion}

In this study, it was found that the plasma endocan levels were higher in non-dipper hypertensive patients than dipper

Table 2. 24-hour ambulatory blood pressure values of dipper and non-dipper groups

\begin{tabular}{lccr}
\hline & Dipper $(\mathbf{n = 3 5 )}$ & Non-dipper $(\mathbf{n = 3 5})$ & $\mathbf{p}$ \\
\hline Daytime SBP $(\mathrm{mmHg})$ & $151 \pm 9$ & $151 \pm 9$ & 0.934 \\
Daytime DBP $(\mathrm{mmHg})$ & $95 \pm 7$ & $94 \pm 10$ & 0.599 \\
Nighttime SBP $(\mathrm{mmHg})$ & $132 \pm 9$ & $147 \pm 11$ & $<0.001$ \\
Nighttime DBP $(\mathrm{mmHg})$ & $80 \pm 7$ & $91 \pm 9$ & $<0.001$ \\
24-h SBP $(\mathrm{mmHg})$ & $147 \pm 8$ & $150 \pm 9$ & 0.120 \\
24-h DBP $(\mathrm{mmHg})$ & $92 \pm 5$ & $93 \pm 9$ & 0.703 \\
\hline
\end{tabular}

All values are presented as mean value and SD. SBP: systolic blood pressure, DBP: diastolic blood pressure

Table 3. Univariable and multivariable logistic regression analysis of associations between non-dipping status and variables in hypertensive patients

\begin{tabular}{|c|c|c|c|c|}
\hline \multirow[b]{2}{*}{ Variables } & \multicolumn{2}{|c|}{ Univariate regression analysis } & \multicolumn{2}{|c|}{ Multivariate regression analysis } \\
\hline & $\begin{array}{c}\text { Beta } \\
\text { (95\% Confidence interval) }\end{array}$ & p & $\begin{array}{c}\text { Beta } \\
\text { (95\% Confidence interval) }\end{array}$ & $\mathbf{p}$ \\
\hline Age & $1.034(0.975-1.097)$ & 0.260 & - & - \\
\hline Female sex & $0.561(0.217-1.449)$ & 0.233 & - & - \\
\hline Smoking & $1.000(0.229-4.361)$ & 1.000 & - & - \\
\hline Hemoglobin & $0.736(0.522-1.038)$ & 0.081 & $0.777(0.532-1.137)$ & 0.194 \\
\hline Fasting glucose & $0.975(0.940-1.011)$ & 0.177 & - & - \\
\hline Creatinine & 0.209 (0.016-2.718) & 0.231 & - & - \\
\hline LVMI & $1.015(0.991-1.040)$ & 0.225 & - & - \\
\hline BMI & $0.787(0.608-1.018)$ & 0.068 & $0.789(0.589-1.056)$ & 0.111 \\
\hline $\mathrm{HsCRP}$ & $1.221(1.045-1.426)$ & 0.012 & $1.201(1.005-1.435)$ & 0.044 \\
\hline Endocan & $1.008(1.003-1.014)$ & 0.004 & $1.007(1.001-1.013)$ & 0.021 \\
\hline
\end{tabular}

BMI: body mass index, LVMI: left ventricular mass index, HsCRP: high-sensitivity C-reactive protein 


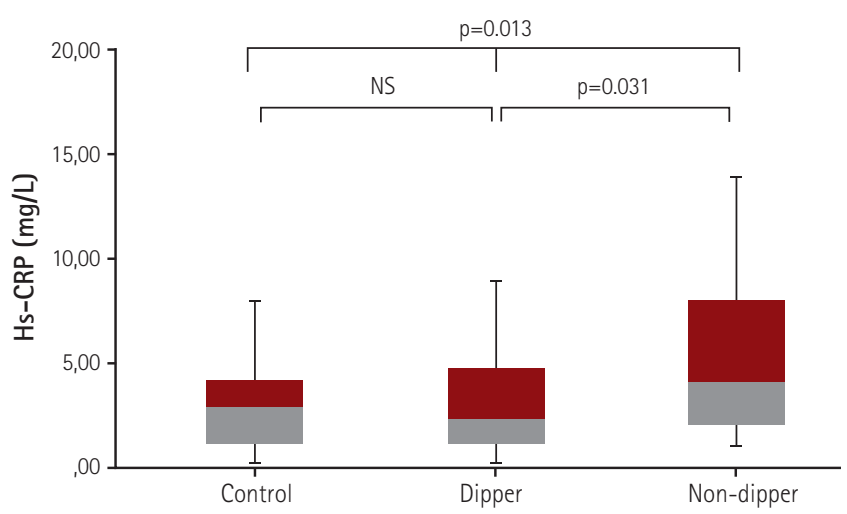

Fig. 2. Comparison of hsCRP levels in non-dippers compared with dippers and controls. NS: non-significant, Hs-CRP: high-sensitivity C-reactive protein.

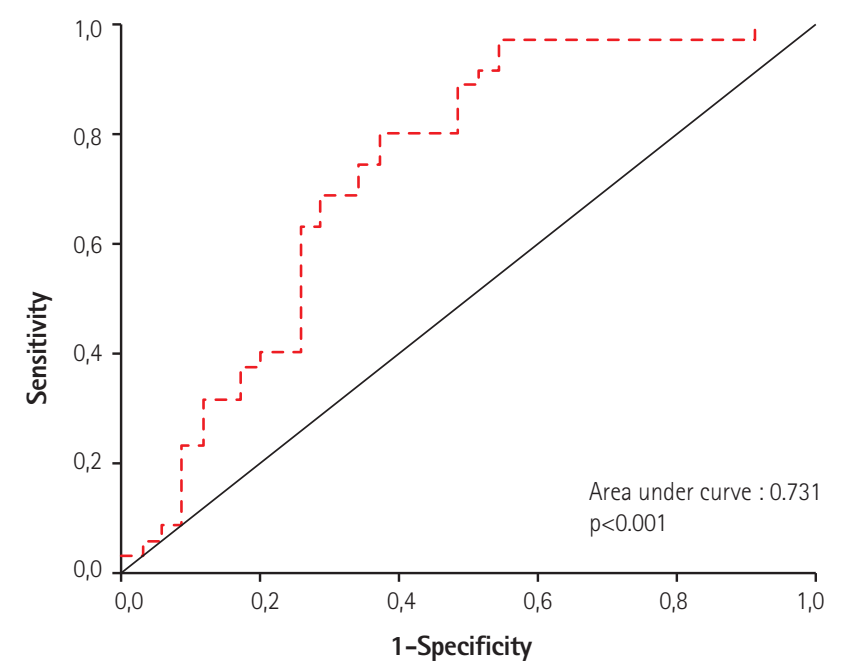

Fig. 3. Receiver operating characteristic curve of endocan for predicting non-dipper hypertension.

hypertensives and normotensives. Further, it was shown that plasma levels of endocan was independently associated with nondipping status. To the best of our knowledge, this study was the first to investigate the relationship between blood levels of endocan and daily circadian BP patterns.

Endocan plays an important role in inflammatory reactions, cellular adhesion, and regulation of tumor progression. ${ }^{1216(17)}$ Expression of endocan increases during inflammation when endothelium is activated by tumor necrosis factor- $\alpha$ (TNF- $\alpha$ ) and interleukin-1 $\beta(\mathrm{IL}-1 \beta)^{16}{ }^{16}$ It has been shown that circulating levels of these cytokines are increased in hypertensive patients. ${ }^{18)}$ Endocan is also elevated in $\mathrm{HT}$ and is correlated with hsCRP. ${ }^{19)}$ It has been demonstrated in studies of Tadzic et al. ${ }^{20)}$ that endocan levels are correlated with ICAM-1, which is induced by pro-inflammatory cytokines such as TNF- $\alpha$ and IL-1 $\beta$ in hypertensive subjects.
Endocan has a key role in the regulation of endothelial dysfunction through promoting endothelium-specific inflammatory disorders. ${ }^{8)}$

A nocturnal non-dipping pattern is a situation accompanied by inflammation ${ }^{21)}$ and endothelial dysfunction. ${ }^{17222}$ The clinical relevance of a non-dipping status in HT patients lies in its proven association with more severe target organ damage and increased risk for cardiovascular events. ${ }^{23224)}$ Many studies showed that left ventricular hypertrophy, carotid intima-media thickness, increased pulse wave velocity and microalbuminuria are much more prevalent in patients with a non-dipping BP pattern. ${ }^{25-27)}$ An endocan level was independently associated with non-dipping pattern in our study. As a result of high inflammatory activity, hsCRP levels were significantly higher than the other groups in non-dippers. HsCRP was also independently associated with non-dipping pattern. Increased endocan levels that is seen in non-dipper HT is considered as reflection of inflammatory activity and endothelial dysfunction. In the present study, the effect of higher night BP levels on endocan could be significant in non-dippers. Despite there was no difference in average 24-hour BP between the group of dippers and non-dippers, elevated hsCRP and endocan levels might be related with increased sympathetic tone. Endothelial dysfunction and autonomic nervous system imbalance often co-exist in the development of $\mathrm{HT}^{2}{ }^{28)}$ Previous studies showed that activation of sympathetic system precludes drop in nocturnal BP and seems to play an important role in endothelial activation. ${ }^{2930)}$ Reduction of BP provides improvement in endothelial dysfunction and also associated with decrement of circulating levels of endocan.719) In our study, as non-dippers are exposed to more endothelial damage due to an overall hypertensive state through the day and night, increased endocan levels may be expected. This and previous findings demonstrate that endocan might be a dynamic biomarker of endothelial activation due to a hypertensive state.

Albeit patients with dipper HT have had higher endocan levels than the control group in patients of our study, it was not shown to have statistical significance. This finding would appear to conflict with previous studies. However, we consider that the reason of this situation might be due to the relatively small number of patients in our study and lack of classification of hypertensive patients in terms of circadian BP pattern in previous studies.

Our study has some limitations, the major limitation being the relatively small sample size. Prospective studies with a larger number of patients are able to clearly state whether endocan predicts high risk hypertensive patients. In addition, the results of our study would be much more robust if endothelial dysfunction was quantified. No usage of pro-inflammatory markers can be counted as another limitation. 


\section{Conclusion}

In conclusion, circulatory levels of endocan were higher in the non-dipper group in comparison with both dippers and normotensives. Additionally, endocan and hsCRP were found to be independently associated with a non-dipping pattern. According to the findings, we suggest that elevated levels of endocan in non-dipper hypertensive patients might be associated with a longer duration of exposure to high BP through the day and night. Therefore, endocan might be a potential marker to quantify endothelial activation in hypertensive patients. These results point to the possible future role of endocan in the selection of hypertensive patients at higher risk or target organ damage.

\section{References}

1. Kannel WB. Blood pressure as a cardiovascular risk factor: prevention and treatment. JAMA 1996:275:1571-6.

2. Panza JA, Quyyumi AA, Brush JE Jr, Epstein SE. Abnormal endothelium-dependent vascular relaxation in patients with essential hypertension. N Engl J Med 1990;323:22-7.

3. Verdecchia P, Schillaci G, Porcellati C. Dippers versus non-dippers. J Hypertens Supp/ 1991;9:S42-4.

4. Verdecchia P, Schillaci G, Borgioni C, et al. Altered circadian blood pressure profile and prognosis. Blood Press Monit 1997;2:347-52.

5. Verdecchia P, Schillaci G, Guerrieri M, et al. Circadian blood pressure changes and left ventricular hypertrophy in essential hypertension. Circulation 1990;81:528-36.

6. Yan $B$, Peng $L$, Han $D$, et al. Blood pressure reverse-dipping is associated with early formation of carotid plaque in senior hypertensive patients. Medicine (Baltimore) 2015;94:e604.

7. Higashi $Y$, Nakagawa $K$, Kimura $M$, et al. Circadian variation of blood pressure and endothelial function in patients with essential hypertension:a comparison of dippers and non-dippers. J Am Coll Cardiol 2002;40:2039-43.

8. Bechard $D$, Meignin $V$, Scherpereel $A$, et al. Characterization of the secreted form of endothelial-cell-specific molecule 1 by specific monoclonal antibodies. J Vasc Res 2000;37:417-25.

9. Bechard D, Scherpereel $A$, Hammad $H_{1}$ et al. Human endothelial-cell specific molecule-1 binds directly to the integrin CD11a/CD18 (LFA-1) and blocks binding to intercellular adhesion molecule-1. J Immunol 2001;167:3099-106.

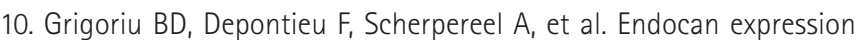
and relationship with survival in human non-small cell lung cancer. Clin Cancer Res 2006;12:4575-82.

11. Abid MR, Yi X, Yano K, Shih SC, Aird WC. Vascular endocan is preferentially expressed in tumor endothelium. Microvasc Res
2006;72:136-45.

12. Balta S, Mikhailidis DP, Demirkol S, et al. Endocan--a novel inflammatory indicator in newly diagnosed patients with hypertension: a pilot study. Angiology 2014;65:773-7.

13. Wang XS, Yang W, Luo T, Wang JM, Jing YY. Serum endocan levels are correlated with the presence and severity of coronary artery disease in patients with hypertension. Genet Test Mol Biomarkers 2015;19:124-7.

14. Lang RM, Badano LP, Mor-Avi V, et al. Recommendations for cardiac chamber quantification by echocardiography in adults: an update from the American Society of Echocardiography and the European Association of Cardiovascular Imaging. J Am Soc Echocardiogr 2015;28:1-39.e14.

15. Mancia G, Fagard R, Narkiewicz K, et al. 2013 ESH/ESC guidelines for the management of arterial hypertension: The Task Force for the Management of Arterial Hypertension of the European Society of Hypertension (ESH) and of the European Society of Cardiology (ESC). Eur Heart J 2013;34:2159-219.

16. Lassalle $P$, Molet $S$, Janin $A$, et al. ESM-1 is a novel human endothelial cell-specific molecule expressed in lung and regulated by cytokines. $J$ Biol Chem 1996;271:20458-64.

17. Sarrazin $S$, Adam $E_{\text {, Lyon } M}$, et al. Endocan or endothelial cell specific molecule-1 (ESM-1): A potential novel endothelial cell marker and a new target for cancer therapy. Biochim Biophys Acta 2006;1765:25-37.

18. Mauno V, Hannu K, Esko K. Proinflammation and Hypertension: A population-based study. Mediators Inflamm 2008;2008:619704.

19. Celik T, Balta S, Karaman M, et al. Endocan, a novel marker of endothelial dysfunction in patients with essential hypertension: Comparative effects of amlodipine and valsartan. Blood Press 2015;24:55-60.

20. Tadzic R, Mihalj M, Vcev A, Ennen J, Tadzic A, Drenjancevic I. The effects of arterial blood pressure reduction on endocan and soluble endothelial cell adhesion molecules (CAMs) and CAMs ligands expression in hypertensive patients on Ca-channel blocker therapy. Kidney Blood Press Res 2013;37:103-15.

21. Tosu AR, Demir $S$, Selcuk $M$, et al. Comparison of inflammatory markers in non-dipper hypertension vs. dipper hypertension and in normotensive individuals: uric acid, C-reactive protein and red blood cell distribution width readings. Postepy Kardiol Interwencyjnej 2014;10:98-103.

22. von Känel $R$, Jain $S$, Mills PJ, et al. Relation of nocturnal blood pressure dipping to cellular adhesion, inflammation and hemostasis. J Hypertens 2004;22:2087-93.

23. Larochelle P. Circadian variation in blood pressure: dipper or nondipper. J Clin Hypertens (Greenwich) 2002;4(4 Suppl 1):3-8.

24. de la Sierra A, Redon J, Banegas JR, et al. Prevalence and factors associated with circadian blood pressure patterns in hypertensive 


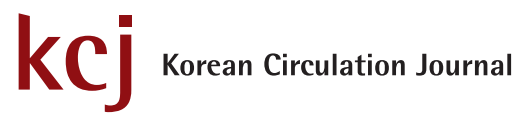

patients. Hypertension 2009;53:466-72.

25. Lurbe $E_{1}$ Redon J, Kesani $A$, et al. Increase in nocturnal blood pressure and progression to microalbuminuria in type 1 diabetes. N Engl J Med 2002;347:797-805.

26. Fan HQ, Li Y, Thijs L, Hansen TW, et al. Prognostic value of isolated nocturnal hypertension on ambulatory measurement in 8711 individuals from 10 populations. J Hypertens 2010;28:2036-45.

27. Ozdemir E, Yildirimturk O, Cengiz B, Yurdakul S, Aytekin S. Evaluation of carotid intima-media thickness and aortic elasticity in patients with nondipper hypertension. Echocardiography 2014;31:663-8.

28. Amiya E, Watanabe M, Komuro I. The Relationship between Vascular Function and the Autonomic Nervous System. Ann Vasc Dis 2014;7:109-19.

29. Millar-Craig MW, Bishop CN, Raftery EB. Circadian variation of blood-pressure. Lancet 1978;1:795-7.

30. Watson RD, Hamilton CA, Reid JL, Littler WA. Changes in plasma norepinephrine, blood pressure and heart rate during physical activity in hypertensive man. Hypertension 1979;1:341-6. 